\title{
Enterobacter cloacae: a villain in CaOx stone disease?
}

\author{
Yuanyuan Yang ${ }^{1} \cdot$ Senyuan Hong ${ }^{1} \cdot$ Jinzhou $\mathrm{Xu}^{1} \cdot$ Cong $\mathrm{Li}^{1} \cdot$ Shaogang Wang ${ }^{1}{ }^{\mathbb{D}} \cdot$ Yang Xun $^{1}$
}

Received: 7 July 2021 / Accepted: 21 January 2022 / Published online: 6 February 2022

(c) The Author(s) 2022

\begin{abstract}
To explore the roles microbiome of urinary tract played in calcium oxalate stones $(\mathrm{CaOx})$ formation, we collected two sides' pelvis urine of patients with unilateral $\mathrm{CaOx}$ stones to set self-control to diminish the influence of systemic factors. Patients with unilateral $\mathrm{CaOx}$ stones were recruited in our study according to strict criteria. 16S rRNA gene sequencing was applied to every pair of pelvis urine. Bacterial genome sequencing of Enterobacter cloacae was conducted and bioinformatic analysis was applied to explore the possible pathways of Enterobacter cloacae inducing $\mathrm{CaOx}$ stones formation. In vivo experiments were conducted to validate our claims. Von Kossa staining, TUNEL assay and Western Blot were applied to SD rats exploring the mechanism of stone formation. We found 26 significantly different bacteria between stone sides and non-stone sides' pelvis urine, among which Enterobacter cloacae ranked the most different. Bacterial genome sequencing of Enterobacter cloacae revealed that its virulence factors included Flagellin, LPS and Fimbrial. GO and KEGG analysis revealed it probably induced $\mathrm{CaOx}$ stone formation via ion binging and signaling transduction pathways. The results of animal experiments indicated that Glyoxylic Acid could promote apoptosis and crystal depositions of kidney comparing with control group while pre-injected with Enterobacter cloacae could apparently compound the effects. While Western Blot demonstrated that Glyoxylic Acid or Enterobacter cloacae could increase the expression of IL-6, Mcp-1, BMP2 and OPN in rats' kidney, Glyoxylic Acid and Enterobacter cloacae together could aggravate these increases. These findings indicated that Enterobacter cloacae might play important roles in $\mathrm{CaOx}$ stones formation. However, this study is just a preliminary exploration; further studies still need to be conducted.
\end{abstract}

Keywords Kidney stone $\cdot$ Microbiome $\cdot$ Enterobacter cloacae $\cdot$ Flagellin

\section{Introduction}

Shaogang Wang

sgwangtjm@163.com

$\checkmark$ Yang Xun

tjxyang1993@163.com

Yuanyuan Yang

TJyangyuanyuan@163.com

Senyuan Hong

westshane@126.com

Jinzhou Xu

jason980620@163.com

Cong $\mathrm{Li}$

licongtjm@163.com

1 Department of Urology, Tongji Hospital, Tongji Medical College, Huazhong University of Science and Technology, Wuhan 430030, Hubei, China
Kidney stone is a common disease with substantial morbidity and high recurrence rate. The prevalence and recurrence rate of stone formation is on the rise worldwide [1]. In China, the incidence of kidney stones is estimated to be $5.8 \%$, including $6.5 \%$ in men and $5.1 \%$ in women [2]. Recurrence of kidney stones can cause severe damage, even renal failure which imposes heavy financial and healthy burden on individuals and society [3]. Recently, instrument miniaturization has greatly improved the surgical treatment of kidney stones. However, medical therapies and prevention have not improved substantially because the mechanism of stone formation remains unclear [4]. About $80 \%$ of kidney stones are composed of $\mathrm{CaOx}$ crystals mixed with varying amounts of calcium phosphate (CaP) [5]. High urinary oxalate is considered to be a crucial risk factor [6], while the urinary calcium is often normal or transient high [7]. So far, how $\mathrm{CaOx}$ stones form still remains unclear. Previous 
studies have focused on systematic factors, such as metabolic or genetic problems [8]. However, both our clinical observation and literature reports indicate that most patients with $\mathrm{CaOx}$ stones have unilateral nidus [9]. And all of these can't be explained by systematic factors. Hence, there may be some local factors in the kidney inducing the formation of $\mathrm{CaOx}$ stones.

Historically, it is widely acknowledged that urinary microbiota is closely related to the formation of infectious stones, bacteria can promote the formation of struvite stones via urease [10]. Recently, with the rapid development of $16 \mathrm{~S}$ rRNA gene sequencing, detection of the microbiome has been widely improved [11]. Thus, increasing evidence shows that bacteria are more broadly associated with kidney stones, not only infectious stones, but also $\mathrm{CaOx}$ stones $[12,13]$. Ryan et al. isolated and purified Escherichia coli and Staphylococcus epidermidis from urine and stones of patients with $\mathrm{CaOx}$ stones, suggesting that the formation of calcium oxalate stone may be related to urinary microbiota [14]. However, the urine samples in this study were collected from bladder of different patients, which cannot eliminate the influence of systemic factors, such as genetics and metabolism factors.

To eliminate the influence of metabolic and genetic abnormalities, we recruited patients with unilateral $\mathrm{CaOx}$ stones and collected their two sides (both the stone sides and the non-stone sides) pelvis urine. We applied 16S rRNA gene sequencing to detect the differences of microbiota between the stone sides and non-stone sides pelvis urine of the same patients. We also performed an in vivo experiment to preliminarily explore the relationship between microbiota and $\mathrm{CaOx}$ stones. This study, to our knowledge, registers the first attempt to disclose the different microbiota of renal pelvis urine between stone side and non-stone side of a same patient, trying to reveal the relationship between urinary microbiota and $\mathrm{CaOx}$ stones.

\section{Methods}

\section{Participants' information and urine samples collection}

Patients came to our department suspected unilateral $\mathrm{CaOx}$ stones which predicted by Computed Tomography were invited to participate in the study. Exclusion criteria included antibiotic exposure prior to 4 weeks, hematuria or other organic diseases. All patients received percutaneous nephro-lithotomy. Pelvis urine derived from each participant were collected at time of percutaneous nephro-lithotomy, after intravenous injection of furosemide, renal pelvis urine was directly collected in an asepsis tube through a ureteral catheter. We collected pelvis urine of both stone sides and non-stone sides. Samples were immediately frozen in liquid nitrogen, and subsequently stored under $-80{ }^{\circ} \mathrm{C}$. The whole process maintained sterility via the instruction of MICROCOSM (an international consortium for microbiome in urinary stone disease) [15]. After stone composition analysis, we selected urine samples of patients with a definite diagnosis of $\mathrm{CaOx}$ stones and applied 16S rRNA gene sequencing. The samples processing and data analysis section were also followed the consortium's instructions to minimize the technical biases and barriers associated with microbiome research. Ethical Review Board of Tongji Hospital, Tongji Medical College, Huazhong University of Science and Technology has approved the collection of renal pelvis urine samples (2021S130). Informed consents were obtained from each participant.

\section{Separation and purification}

Enterobacter cloacae isolates were collected from pelvis urine of patients recruited in our study using Enterobacter Cloacae Isolation Agar (SoleyBio, Beijing, QP0382). Whole Genome Sequencing (WGS) was used to confirm the Enterobacter cloacae and explore the characteristics of it.

\section{5 rRNA gene sequencing and Whole Genome Sequencing (WGS)}

Total genomic DNAs were extracted using the OMEGA Soil DNA Kit (D5625-01) (Omega Bio-Tek, Norcross, GA, USA) and stored at $-20{ }^{\circ} \mathrm{C}$ prior to further analysis. PCR amplification of the nearly full-length bacterial 16S rRNA genes was performed using the forward primer $27 \mathrm{~F}$ ( $5^{\prime}$-AGA GTTTGA TCMTGGCTCAG-3') and the reverse primer 1492R (5'-ACCTTGTTACGACTT-3'). The extracted DNA's amplification was conducted via two-step PCR, with samplespecific 16-bp barcodes incorporated into the forward and reverse primers in the second PCR step. After quantification, amplicons were pooled in equal amounts, and Single Molecule Real-Time (SMRT) sequencing was performed using the PacBio Sequel platform. Raw sequences were elaborated through the PacBio SMRT Link portal (version 5.0.1.9585). Microbiome bioinformatics were performed with QIIME2 (https://docs.qiime2.org/2019.4/tutorials/) [16]. Raw sequence data were de-multiplexed and qualityfiltered, de-noised, merged and chimera removed using the DADA2 plugin [17]. Taxonomy was assigned to amplicon sequence variants (ASVs) using the classify-sklearn naïve Bayes taxonomy classifier in feature-classifier plugin against the SILVA Release 132 Database [18, 19]. 


\section{Bioinformatics and statistical analysis}

Sequence analysis was mainly performed using QIIME2 and R packages (v3.2.0). Alpha diversity indices, such as Chao1, Simpson index, Shannon index, Pielou's evenness, Observed species, Faith's PD and Good's coverage, were calculated using the ASV table in QIIME2, and visualized as box plots. Beta diversity analysis was conducted to explore the structural variation of microbial communities across Hea and Lith groups using unweighted uniFrac distance metrics [20], visualized as principal coordinate analysis (PCoA) and evaluated by the Adonis test [21]. Venn diagram was generated to visualize the common and unique ASVs among Hea groups and Lith groups via R package "Venn Diagram" [22]. Linear discriminant analysis Effect Size (LEfSe) analysis was used to identify differentially abundant bacteria among the Hea groups and Lith groups with a cutoff of 2.0. PICRUSt2 package was used to conduct the Kyoto Encyclopedia of Genes and Genomes (KEGG) pathways analysis, Gene Ontology analysis and Metacyc pathways analysis.

\section{Animal experiment design}

Twelve male SD rats ( 8 weeks old, $300 \mathrm{~g}$ ) were purchased from the experimental Animal Centre of Tongji Hospital, Tongji Medical College, Huazhong University of Science and Technology. All procedures were approved by the Animal Care and Use Committee of Tongji Hospital, Tongji Medical College, Huazhong University of Science and Technology. Rats were acclimatized to the environment of $12 \mathrm{~h} \mathrm{light/dark} \mathrm{cycle} \mathrm{for} 1$ week in a specific pathogen-free animal house. Rats were randomly divided into the following four groups of 3 rats each: control group; Glyoxylic Acid group, intraperitoneally injected with Glyoxylic Acid $(10.5 \mathrm{mg} / \mathrm{ml}, 6.66 \mathrm{ml} / \mathrm{kg}$, MackLin, Shanghai) every day for nine days; Enterobacter cloacae group, renal pelvis injection of Enterobacter cloacae $\left(2 * 10^{8} \mathrm{cfu} / \mathrm{ml}, 50 \mathrm{ul}\right)$; Glyoxylic Acid + Enterobacter cloacae group, renal pelvis injection of Enterobacter cloacae $\left(2 * 10^{8} \mathrm{cfu} / \mathrm{ml}, 50 \mathrm{ul}\right)$, then intraperitoneally injected with Glyoxylic Acid $(10.5 \mathrm{mg} / \mathrm{ml}, 6.66 \mathrm{ml} /$ $\mathrm{kg}$, MackLin, Shanghai) every day for nine days. All rats were euthanized after the above treatment for 2 weeks. The renal tissues were collected, with fixed with $4 \%$ paraformaldehyde or frozen at $-80^{\circ} \mathrm{C}$ for further use.

\section{Detection of crystal deposition by Von Kossa staining and apoptosis by TUNEL Assay}

Apoptoses of the kidney tissues were assessed with TUNEL assay, following the manufacturer's instructions (Vazyme). Images were captured using a BX53 fluorescence microscope (Olympus, Tokyo, Japan). The crystal depositions in the kidneys were analyzed by Von Kossa staining according to manufacturer's instructions (Solarbio, Beijing). The stained tissues were observed by microscopy (Olympus, Japan).

\section{Western blot analysis}

The kidney tissues were cut up and lysed in RIPA Lysis Buffer with protease inhibitor phenylmethanesulfonyl fluoride (PMSF) and phospho-proteinase inhibitors (Beyotime Biotechnology, Shanghai, China). The protein concentration was measured using the bicinchoninic acid (BCA) protein assay kit (Beyotime Biotechnology, Shanghai, China). Proteins were separated and isolated using sodium dodecyl sulfate-polyacrylamide gel electrophoresis with $5 \%$ and $12 \%$ for $120 \mathrm{~V}, 2 \mathrm{~h}$ and then transferred onto polyvinylidene fluoride (PVDF) membranes for $200 \mathrm{~mA}, 70 \mathrm{~min}$ (MCP-1, IL-6) and $200 \mathrm{ma}, 90 \mathrm{~min}$ (BMP2, OPN). The PVDF membranes were blocked with 5\% bovine serum albumin for $2 \mathrm{~h}$. The membranes were incubated with primary antibody against BMP2 (proteintech group, 66,383-1-Ig, China, 1:1500), OPN (Boster, PB0589, China, 1:1000), IL-6 (Boster, BA4399, China, 1:1000), MCP-1 (proteintech, 66,272-1Ig, China, 1:1500), $\beta$-actin (proteintech, 66,009-1-Ig, China, $1: 4000)$ at $4{ }^{\circ} \mathrm{C}$ overnight. Then they were incubated with secondary antibody at room temperature for $2 \mathrm{~h}$, the proteins were visualized using enhanced developer (Boster, China). The gray values of these proteins were analyzed with imagepro plus. All the WB quantifications were used three independent Western blot results.

\section{Results}

\section{General characteristics of patients with $\mathrm{CaOx}$ stones}

Patients with unilateral $\mathrm{CaOx}$ stones were involved in this study. Finally, we brought 4 patients, that is to say, 8 samples into our study according to our strict inclusion criteria. Detailed information of participants was listed in Table 1. These kidney stones were primarily composed of calcium oxalate. The calcium concentration of these participants was normal. The 16S rRNA gene sequencing was applied to both the stone side and non-stone side pelvis urine of every participant to eliminate systemic factors like metabolic and genetic factors.

\section{Richness and diversity of the pelvis urine microbiota in stone sides and non-stone sides}

The pelvis urine derived from both two sides of each participant was applied to 16S rRNA gene sequencing analysis. A total of 103,198 high-quality sequences were obtained from these 8 samples with an average length of 1462 . All the 
Table 1 Basic information of patients included in this study

\begin{tabular}{lllllll}
\hline ID & Age $(\mathrm{y})$ & Gender & BMI $\left(\mathrm{kg} / \mathrm{m}^{2}\right)$ & $\begin{array}{l}\text { Plasma Ca } \\
(\mathrm{mmol} / \mathrm{L})\end{array}$ & Stone side & Stone type \\
\hline 1 & 68 & Female & 23.8 & 2.31 & Right & $\mathrm{CaOx}$ \\
2 & 42 & Male & 30 & 2.25 & Left & $\mathrm{CaOx}$ \\
3 & 42 & Male & 27.9 & 2.33 & Left & $\mathrm{CaOx}$ \\
4 & 60 & Male & 26.3 & 2.2 & Right & $\mathrm{CaOx}$ \\
\hline
\end{tabular}

$\mathrm{CaOx}$ Calcium oxalate sequences were clustered into 1027 ASVs, which belonged to 794 genera and 58 phyla. The Kruskal-Wallis test showed that alpha diversity indices including Chao1, Simpson, Shannon and Observed species indicated no significant difference in within-habitat diversity (Fig. 1a). PCoA at the ASV level showed that the overall microbiota composition of the Lith (stone side) and Hea (non-stone side) groups were different, In other words, between-habitats diversity was different, which was confirmed by the adonis test $(P<0.01)$ (Fig. 1b).

\section{Taxonomic analysis of microbiota composition between stone sides and non-stone sides of unilateral $\mathrm{CaOx}$ stone patients}

Venn diagram showed that Lith and Hea groups shared only 138 (8.16\%) ASVs in common, 1228 (72.62\%) ASVs were identified only in Lith group and 325 (19.22\%) only in Hea group (Fig. 2a). Taxonomic assignment of the ASVs revealed the composition of the bacterial population down to the phylum and genus level. At the phylum level, Proteobacteria was the most common bacteria in Lith groups, followed by Firmicutes. At the genus level, the common bacteria of Lith groups were Pseudomonas, Acinetobacter, Klebsiella and Escherichia (Fig. 2b). LDA effect size analysis (LEfSe) showed the classification hierarchy relationship of the main classification units from phylum to species (from inner to outer ring) in the sample community. From this cladogram, we found that 11 bacteria were stably rich in Lith groups at species level, which might play important roles in $\mathrm{CaOx}$ stones (Fig. 2c). To further compare the differences in species composition among samples and display the distribution trend of species abundance in each sample, we used a heatmap for species composition analysis. According to the screening bacteria $(P<0.05)$, we identified 26 most significantly different bacteria at species level. Detailed information of these 26 bacteria was shown in Table 2. Among these dysregulated bacteria, Enterobacter cloacae and Chryseobacterium sp. were the most stably up-regulated bacteria in stone side of unilateral a

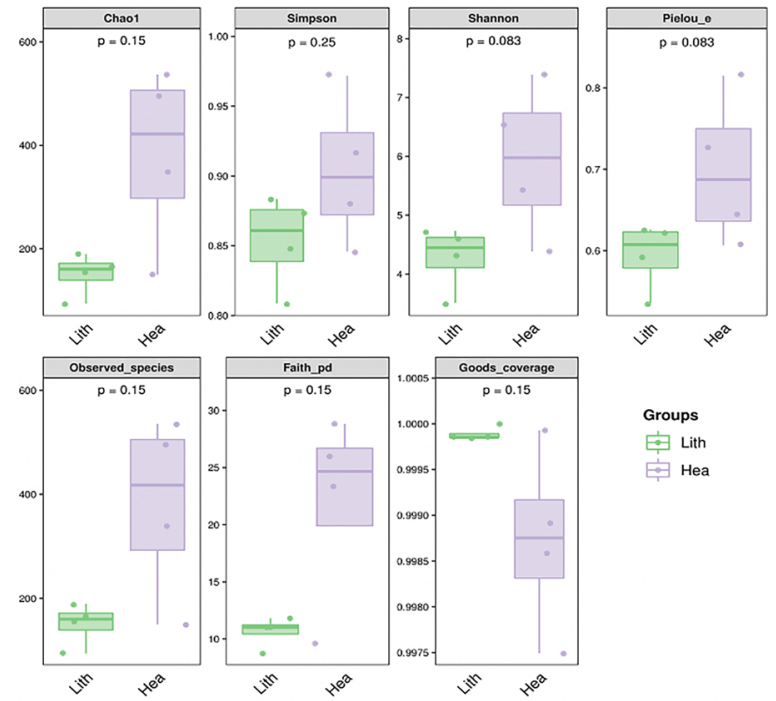

Fig. 1 Richness and diversity of the pelvis urine microbiota in stone sides and non-stone sides. a Comparison of alpha diversity of pelvis urine microbiota among both non-stone sides (Hea) and stone sides (Lith). Chao1, Shannon, Simpson, Pielou_e, observed species, Faith_ pd and Goods coverage indices at amplicon sequence variants (ASVs) level were compared between both non-stone sides (Hea) and stone

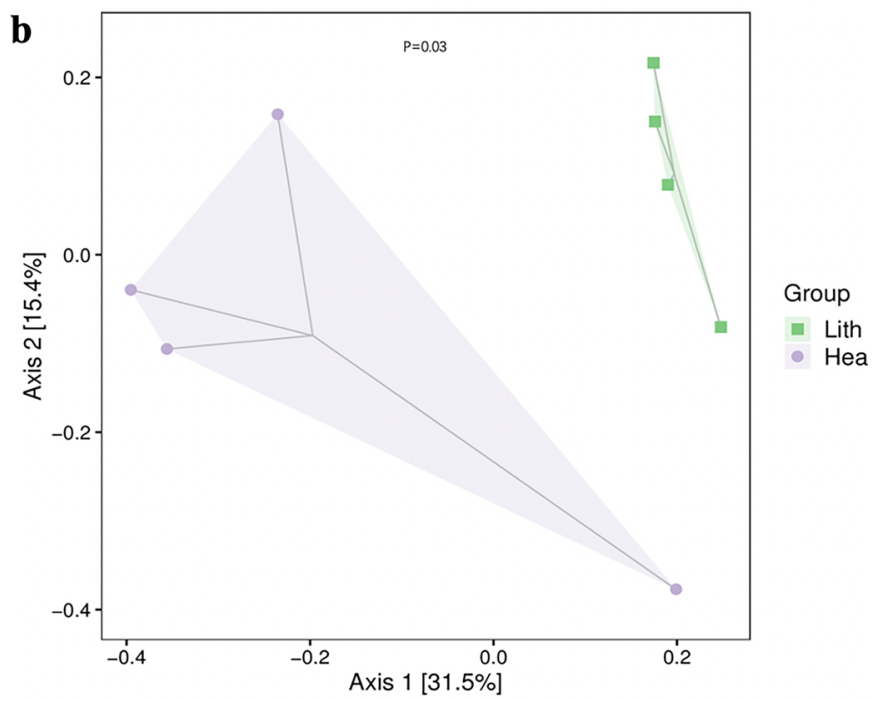

sides (Lith) by the Kruskal-Wallis test. b Comparison of beta diversity of pelvis urine microbiota among both non-stone sides (Hea) and stone sides (Lith). Beta diversity is conducted via unweighted unifrac-based PCoA, PCoA scatter plot based on binary Pearson distance at ASVs level revealed classification of non-stone sides and stone sides 

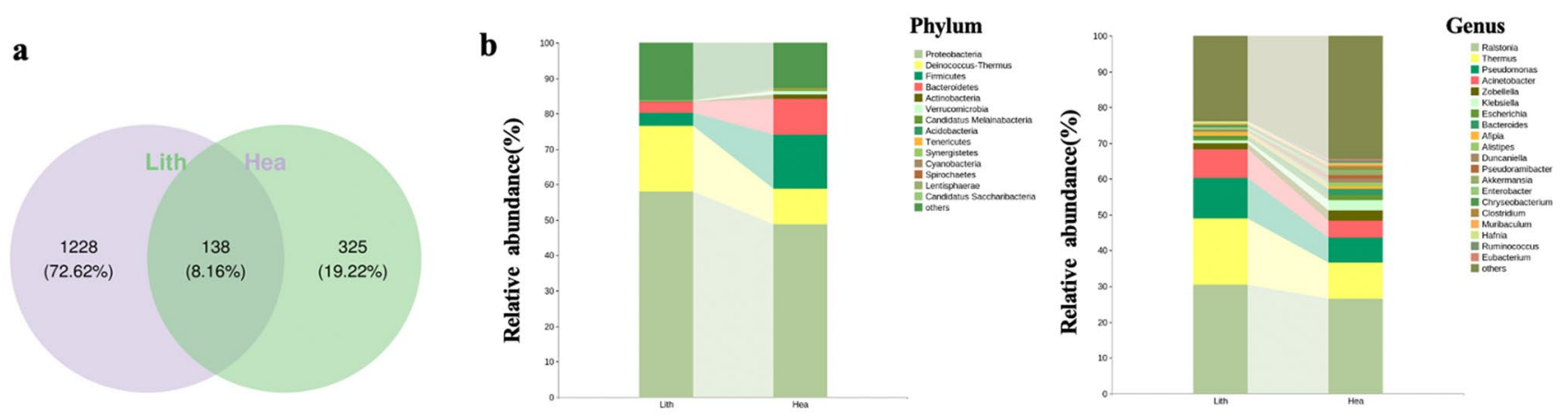

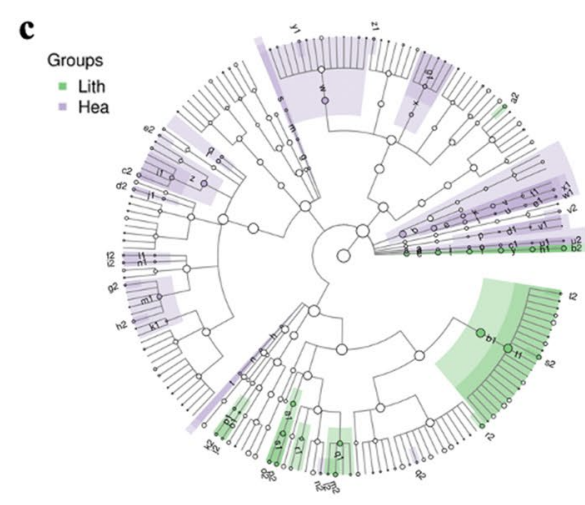

Fig. 2 Taxonomic analysis of microbiota composition between stone sides and non-stone sides of unilateral $\mathrm{CaOx}$ stone patients. a A total of 1691 bacteria in pelvis urine were identified, of which 1228 were found in stone sides and 325 in non-stone sides. b The composition of the microbiota in Hea and Lith group at phylum or genus level. c The cladogram shows the classification hierarchy relationship of the main classification units from phylum to species (from inner to outer ring) in the sample community. The node size corresponds to the average relative abundance of the classification unit, and the hollow node represents a classification unit with no significant differences between groups, while solid nodes indicate significant intergroup

\section{Whole Genome Sequencing (WGS) of Enterobacter cloacae isolated and purified from pelvis urine}

To further explore Enterobacter cloacae, we conducted Whole Genome Sequencing of Enterobacter cloacae isolated and purified from pelvis urine. Genomic Graph made a comprehensive view of the Enterobacter cloacae, including plasmid and chromatin (Fig. 5). Furthermore, we performed GO and KEGG analysis to find possible pathways that Enterobacter cloacae utilized to promote stones formation. Bioinformatics results suggested that ion binding and signal transduction pathways are enriched. Given that $\mathrm{CaOx}$ stones are closely associated with calcium ions and signal transduction, Enterobacter cloacae may be a possible breakthrough for $\mathrm{CaOx}$ stone formation (Fig. 6). What's more, we explored the possible virulence factors of Enterobacter cloacae and found proteins associated with flagellum were the primary factors (Table 3 ). 
Table 2 Basic information of 26 significantly different bacteria

\begin{tabular}{|c|c|c|c|c|c|c|}
\hline Taxa (species) & Abundance & LDA score & $P$ value & Gram staining & Group & Characteristic description \\
\hline Thermus thermophilus & 5.264 & 4.610 & 0.021 & Negative & Stone & PHA synthesis \\
\hline Acinetobacter sp. & 4.281 & 3.76 & 0.021 & Negative & Stone & $\begin{array}{l}\text { Biodegradation } \\
\text { Bioremediation }\end{array}$ \\
\hline Pseudomonaslundensis & 4.250 & 3.706 & 0.021 & Negative & Stone & Hospital Infection \\
\hline Pseudomonas weihenstephanensis & 2.845 & 3.559 & 0.047 & Negative & Stone & Hospital Infection \\
\hline Enterobacter asburiae & 2.743 & 3.459 & 0.047 & Negative & Stone & $\begin{array}{l}\text { Aid plant growth } \\
\text { Degrade polyethylene }\end{array}$ \\
\hline Enterobacter cloacae & 3.838 & 3.459 & 0.021 & Negative & Stone & Catalase Positive \\
\hline Herbaspirillum huttiense & 2.967 & 3.415 & 0.038 & Negative & Stone & Bacteremia/RTI \\
\hline Acidovorax sp. & 2.947 & 3.322 & 0.047 & Negative & Stone & Degrade Thiobencarb \\
\hline Hafnia paralvei & 3.644 & 3.290 & 0.043 & Negative & Stone & Gastroenteritis \\
\hline Chryseobacterium sp. & 3.521 & 3.219 & 0.020 & Negative & Stone & Degrade DDT \\
\hline Hafnia alvei & 3.390 & 3.109 & 0.042 & Negative & Stone & Urosepsislcarcinoma of colon and rectum \\
\hline Alistipes sp. & 3.916 & 3.707 & 0.047 & Negative & Control & Ulcerative Colitis \\
\hline Akkermansia glycaniphila & 3.987 & 3.702 & 0.047 & Negative & Control & Degrade Mucoprotein \\
\hline Clostridium sp. & 3.883 & 3.534 & 0.021 & Positive & Control & Gut microbiota \\
\hline Ruminococcus sp. & 3.677 & 3.419 & 0.014 & Positive & Control & Association with ASD \\
\hline Rhabdanaerobium thermarum & 3.489 & 3.292 & 0.014 & Positive & control & Isolated from a hot spring \\
\hline Ruminococcus albus & 3.301 & 3.248 & 0.047 & Positive & control & Association with ASD \\
\hline Bacteroides sp. & 3.464 & 3.232 & 0.047 & Negative & control & Opportunistic Infection \\
\hline Acinetobacter bereziniae & 3.213 & 3.173 & 0.047 & Negative & control & Opportunistic Infection \\
\hline Oscillibacter valericigenes & 3.400 & 3.158 & 0.047 & Positive & control & $\begin{array}{l}\text { Isolated from the alimentary canal of a } \\
\text { Japanese corbicula clam }\end{array}$ \\
\hline Neglecta timonensis & 2.814 & 3.138 & 0.047 & Positive & control & Type 2 Diabetes \\
\hline Olsenella sp. & 3.072 & 3.125 & 0.047 & Negative & control & Gut microbiota \\
\hline Jonquetella anthropi & 2.471 & 3.119 & 0.047 & Negative & control & Oxidase- and catalase-negative \\
\hline Klebsiella quasipneumoniae & 3.217 & 3.080 & 0.047 & Negative & control & bacterial hepatic abscess \\
\hline Ruthenibacterium lactatiformans & 2.991 & 3.074 & 0.047 & Negative & control & Induce CD8 + T \\
\hline Slackia faecicanis & 2.800 & 3.063 & 0.047 & Positive & control & catalase- and oxidase-negative \\
\hline
\end{tabular}

PHA Polyhydroxylalkanoate, RTI Respiratory tract infection, ASD Autistic Spectrum Disorder

\section{Effects of Enterobacter cloacae on the crystal deposition and apoptosis}

Apoptosis was assessed using Terminal Deoxynucleotidyl Transferase dUTP Nick-End Labeling (TUNEL) assays. Rats injected Glyoxylic Acid with pre-injection of Enterobacter cloacae into renal pelvis expressed severer apoptosis than rats only injected Glyoxylic Acid or Enterobacter cloacae (Fig. 7a). Furthermore, Crystal depositions were examined using von Kossa staining. Crystal depositions of rats injected Glyoxylic Acid with pre-injection of Enterobacter cloacae into renal pelvis were apparently severer than rats only injected Glyoxylic Acid or Enterobacter cloacae (Fig. 7b).

\section{Effects of Enterobacter cloacae on the expression of BMP2, OPN, IL-6 and MCP-1}

Western blot analysis indicated that the expression of inflammation-associated proteins IL-6, MCP-1 and osteoblast-associated protein BMP2 and osteopontin (OPN) notably increased in rats injected Glyoxylic or Enterobacter cloacae compared with that in control rats, and these increases were significantly enhanced when these rats had been treated with both Enterobacter cloacae and Glyoxylic Acid (Fig. 7c).

\section{Discussion}

The components of kidney stones can be classified into following types: calcium oxalate, calcium phosphate, struvite, purine, or cystine. Infection has long been considered to be only associated with struvite, most bacteria present in these stones, such as Proteus species, Klebsiella, Pseudomonas and Corynebacterium species, promote the formation of struvite stones via urease [10]. However, struvite stones account for only $4 \%$ of all urinary stones [23]. Bacteria have shown to be more broadly associated with kidney stones, 
a

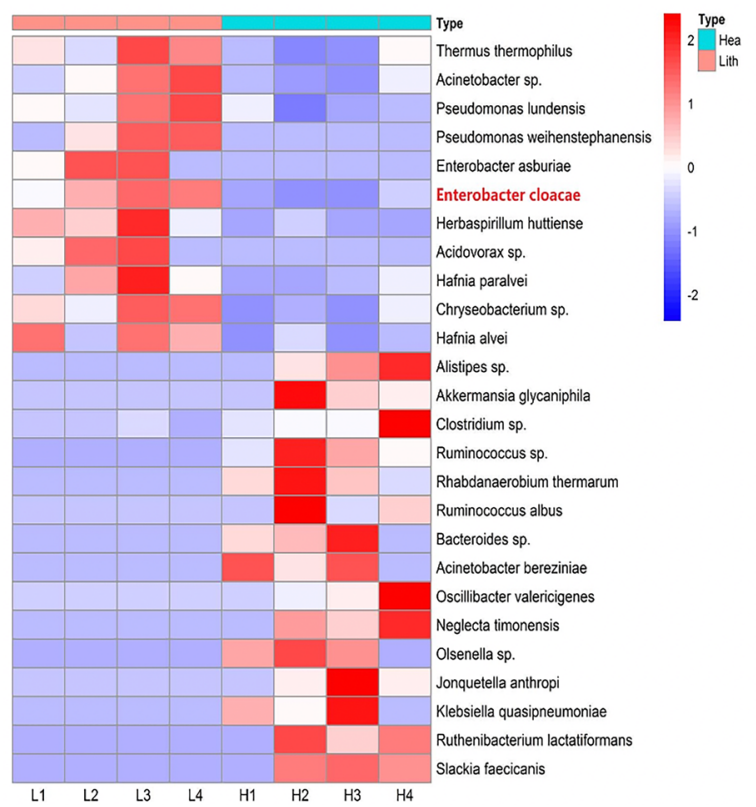

b
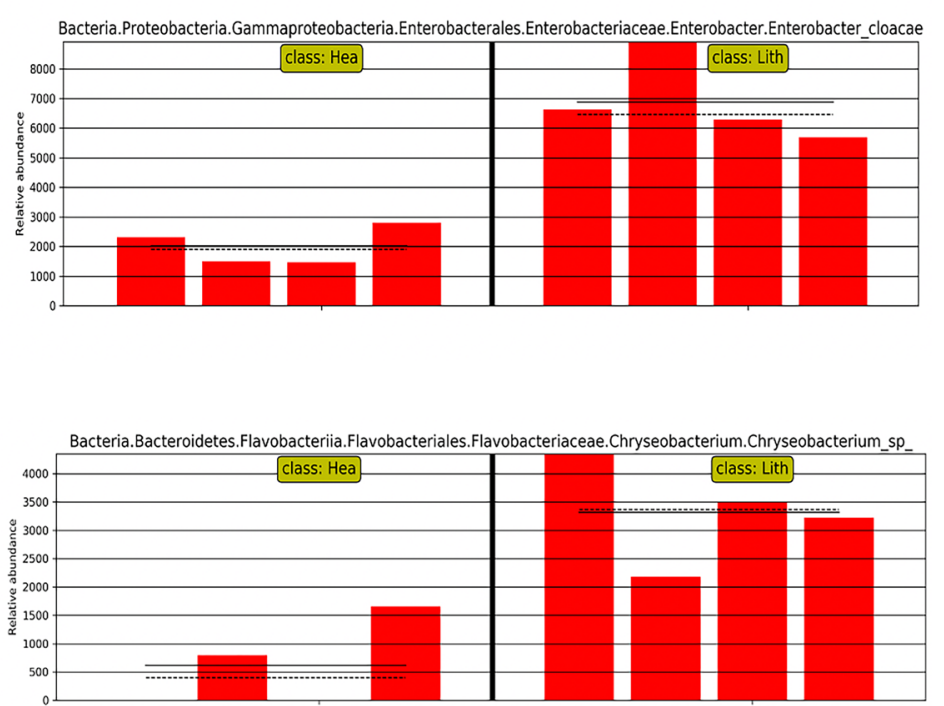

of Enterobacter cloacae and chryseobacterium sp. in pelvis Urine of both stone sides (Lith) and non-stone sides (Hea). The scale of $y$-axis is the original relative abundance times $1,000,000$ to include some very low abundance
Fig. 3 The different bacteria between non-stone sides (Hea) and stone sides (Lith). a With the screening criteria of $p<0.05,26$ species of bacteria were found to be most significantly different between two sides. The scale of red-blue spectrum represented the abundance after zero-mean normalization ( $Z$-score). b The relative abundance a

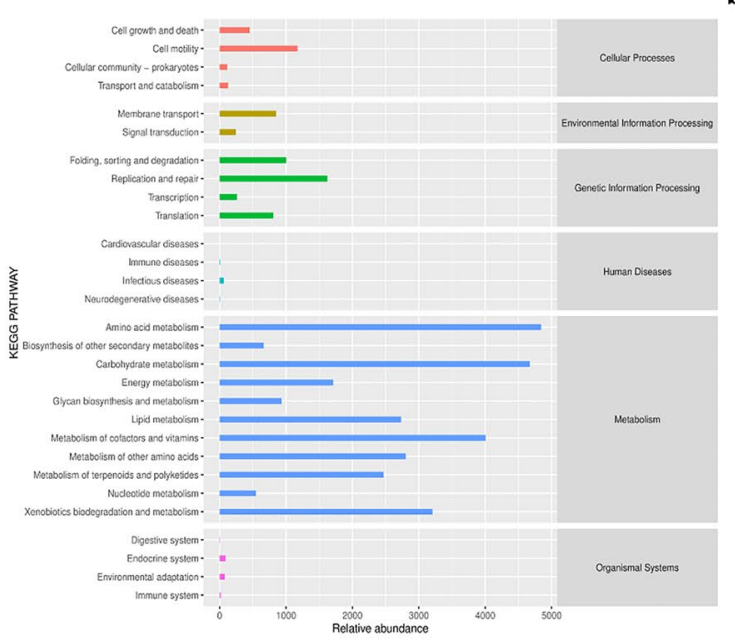

b
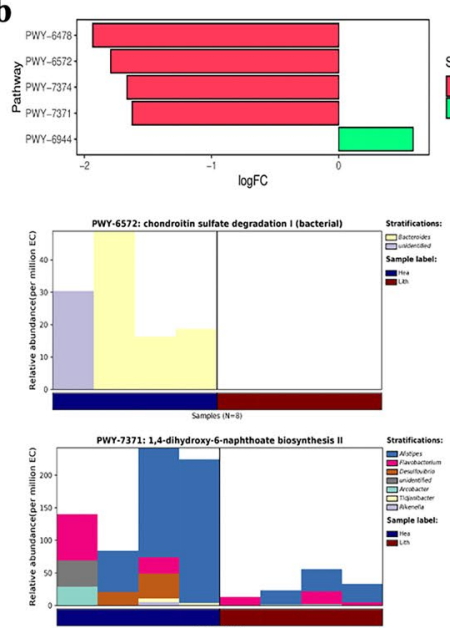
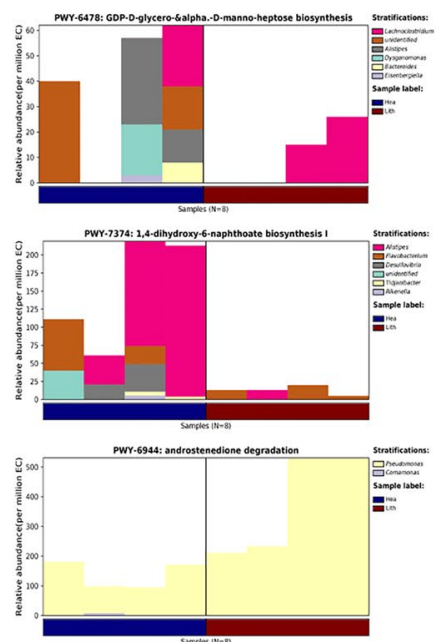

Fig. 4 Bioinformatics analysis of microbiota of pelvis urine. a KEGG pathways analysis of pelvis Urine of both stone sides and non-stone sides. The unit of the $x$-axis is KO per $1,000,000$. b The different

patients with kidney stones often present with concomitant urinary tract infection, regardless of stone composition [24]. The majority of urinary stones are calcium oxalate combined with apatite. Previous studies of $\mathrm{CaOx}$ stones used to focus on metabolic and genetic factors. However, kidney stones are
MetaCyc pathways between stone sides and non-stone sides pelvis Urine of patients with $\mathrm{CaOx}$ stones

often unilateral which cannot be explained by such systematic factors. There may exist some local factors contributing to stones formation. What's more, some urease-negative organisms, for example, Escherichia coli, have been found in calcium-based stones. Therefore, bacteria may be associated 


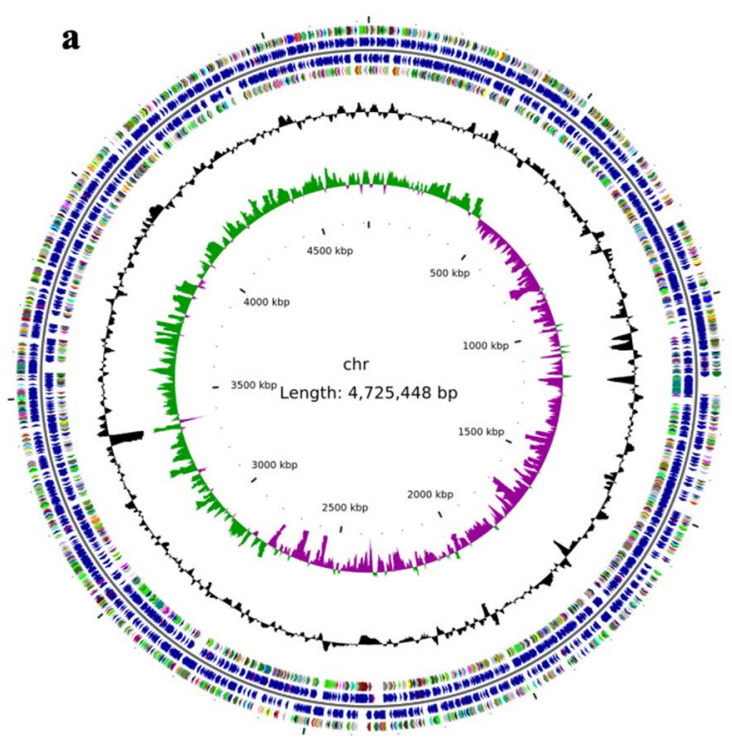

Fig. 5 Genomic Graph of Enterobacter cloacae. a Genomic Graph of chromosome. b Genomic Graph of plasmid. From inside to outside, the first circle represents the scale; the second circle represents GC Skew; the third circle represents GC content; the fourth and seventh

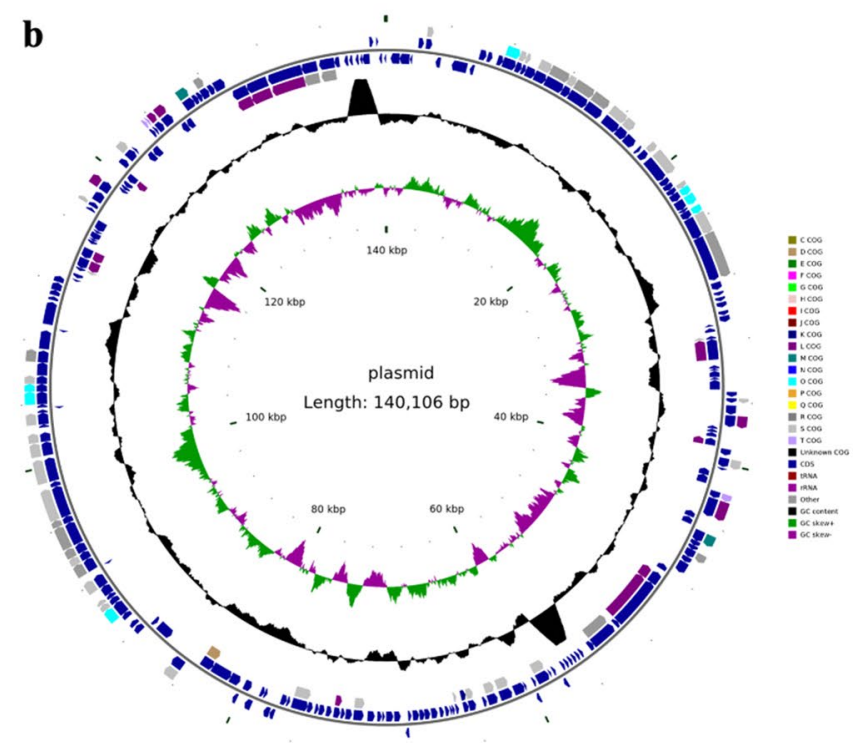

circles represent the COG to which each CDS belongs; the fifth and sixth loops represent the positions of CDS, tRNA, and rRNA on the genome
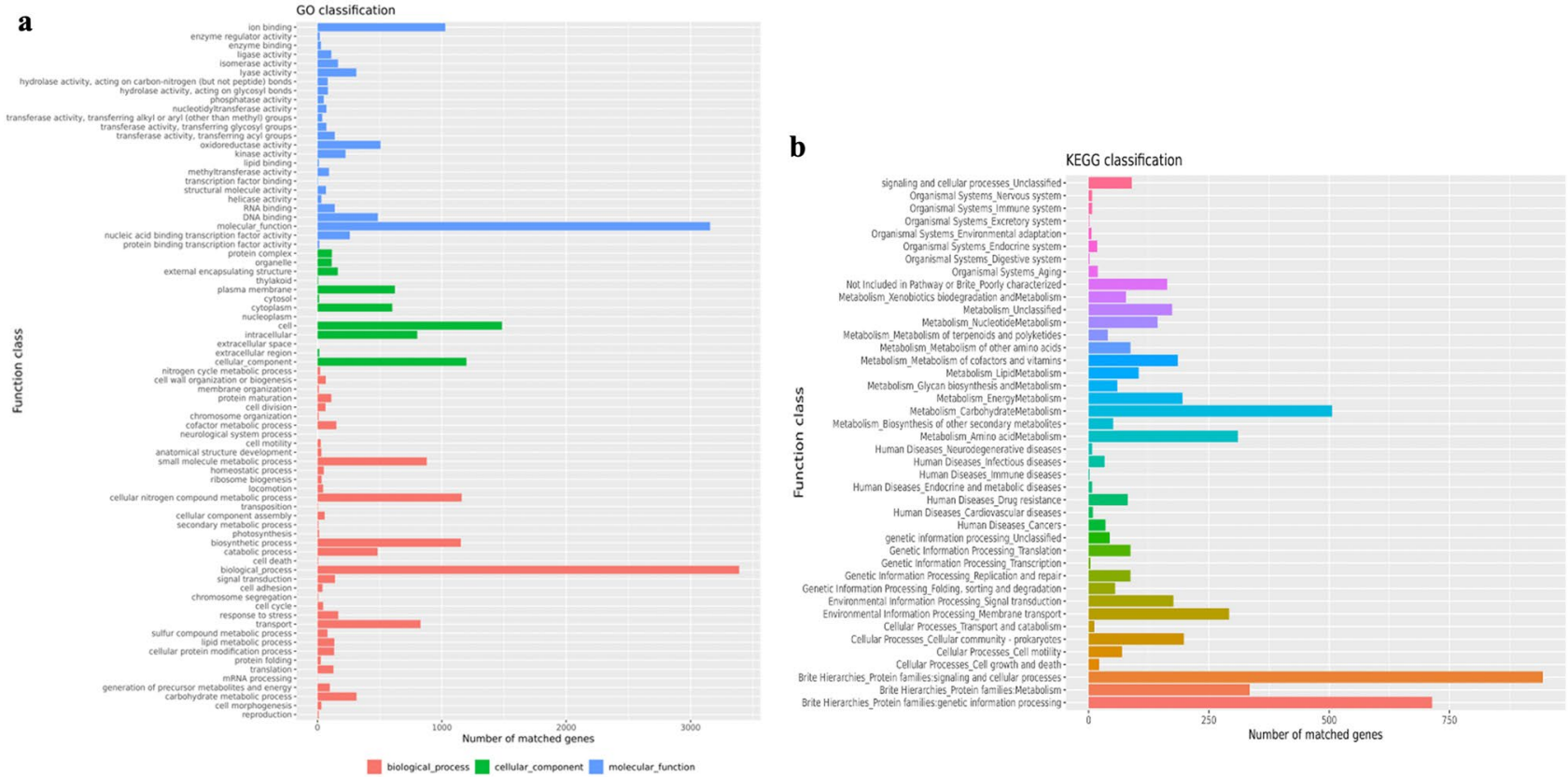

Fig. 6 Bioinformatics analysis of Enterobacter cloacae. a Gene oncology analysis of Enterobacter cloacae. b KEGG pathways analysis of Enterobacter cloacae

with $\mathrm{CaOx}$ stones [14]. Though many studies have revealed the association, the role bacteria playing in $\mathrm{CaOx}$ stones formation remains poorly understood.

Many studies have explored the microbiome of calciumbased urinary stones. Barr-Beare $\mathrm{E}$ and his colleague found the dominant bacterial communities of $\mathrm{CaOx}$ stones including Enterobacteriaceae and the genera Pseudomonas [25]. Ryan et al. added to these bacterial communities with additional identification of Staphylococcus, Veillonella, Streptococcus, Corynebacterium, Haemophilus, Lactobacillus, and 
Table 3 Virulence factors of Enterobacter cloacae

\begin{tabular}{lll}
\hline VF-Name & Symbols VF & VF-Full Name \\
\hline Flh & FlhA,FlhB,FlhC,FlhD & Flagellar biosynthesis protein \\
Che & cheA,cheB,cheW,cheY,cheZ & Chemotaxis protein \\
Fli & fliA,fliF,fliG,fliH,flil,fliN,fli,fliQ,fliR,fliS & Flagellar biosynthetic protein \\
mot & motA,motB & Flagellar motor protein \\
flg & flgB,flgC,flaD,flaE,flgF,flgG,flgH,flgl & Flagellar basal-body rod protein \\
gtr & gtrA,gtrB & Bactoprenol glucosyl transferase \\
acp & acpXL & Acyl carrier protein \\
bcf & bcfA,bcfB,bcfC & Fimbrial subunit \\
fep & fepA,fepB,fepC,fepD,fepG & Ferrienterobactin transporter \\
ure & ureB,ureG & Urease protein \\
\hline
\end{tabular}

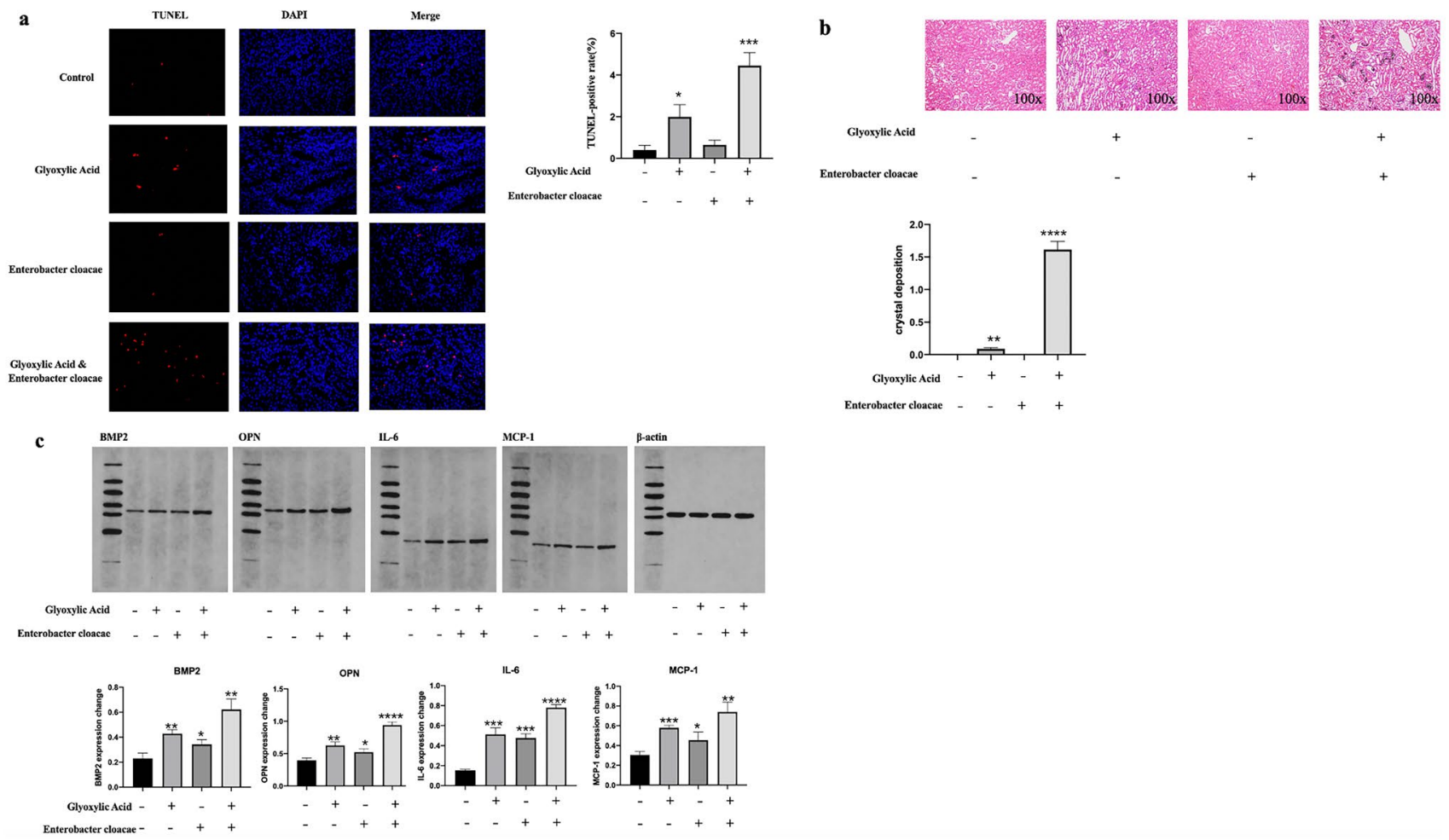

Fig. 7 Effects of Enterobacter cloacae on the pathophysiological changes in the renal of SD rats. a Representative micrographs showed TUNEL staining images of different groups from indicated rats to assess apoptosis. The data are expressed as mean \pm SE. $* P<0.05$ compared with the control group. b Von Kossa staining to detect crystal deposition in the rat kidneys, Glyoxylic Acid and Enterobacter cloacae aggravated the crystal depositions compared with other groups. The data are expressed as mean \pm SE. $* P<0.05$ compared

Bifidobacterium [14]. However, there are still many limitations in previous studies. Most studies collected stones or bladder urine though patients only have one-side stones, microbiota of stones or bladder urine are a mixture of two sides upper urinary tracts which cannot represent the difference between the stone sides and non-stone sides kidney. To replenish the gap, we collected pelvis urine of both with the control group. c Western blot assay showed that the expression levels of BMP2, OPN, IL-6, MCP-1 increased in the kidney tissues in the Glyoxylic Acid or Enterobacter cloacae groups compared with those in the control group. Glyoxylic Acid and Enterobacter cloacae together could apparently enhance these effects. Western blot bands were quantified using ImageJ software. The data are expressed as mean \pm SE. $* P<0.05$ compared with the Control group

stone sides and non-stone sides of patients with unilateral $\mathrm{CaOx}$ stones to make self-control. Thus, we can diminish the influence of systemic factors and explore the local factors which relate to $\mathrm{CaOx}$ stones. Moreover, patients' antibiotic exposures prior to 4 weeks were not tracked in case antibiotics destroy the bacteria initially colonized [26]. Nextgeneration 16S rRNA gene sequencing was conducted to the 
pelvis urine collected from both sides of patients' kidney, the results can be accurate to the species level; thus, we can have a clear cognition of the difference between stone sides and non-stone sides kidneys.

Through the 16S rRNA gene sequencing of urine samples, we found there were significant differences in 26 species of bacteria in bilateral pelvis urine, these bacteria were enriched in PWY-6478, PWY-6572, PWY-7374, PWY-6944 (MetaCyc database), the bacteria enriched in stone sides pelvis urine were mostly enriched in PWY-6944 pathway (androstenedione degradation). Androstenedione is an androgen, urinary androgens are significantly associated with urinary calcium and citrate excretion, which will finally lead to stones formation [7]. What's more, among the 26 differentially expressed bacteria in bilateral pelvis urine, Enterobacter cloacae and Chryseobacterium sp. were most enriched on the stone sides' pelvis urine. Chryseobacterium $\mathrm{sp}$. is a phosphate-solubilizing bacteria (PBS), it was often isolated from soil [27]. At present, the studies on it mostly focus on agriculture. What's more, Ryan A. Dornbier and his colleagues have found that Enterobacter cloacae was isolated from $\mathrm{CaOx}$ stones with concordant enrichment on $16 \mathrm{~S}$ rRNA gene sequencing. Thus, we did not pay much attention to chryseobacterium. Meanwhile, the exploration of Enterobacter cloacae has increased yearly. Enterobacter cloacae is important symbiotic bacteria with intestine, belonging to Enterobacter genus, Enterobacter family, Enterobacter order. It usually exists in the intestinal tract of $40-80 \%$ human body, and can also exist in the respiratory and urinary tract, causing urinary and respiratory tract infections [28]. Enterobacter cloacae has strong adhesion and weak invasion, and can play a role by rearranging cytoskeleton proteins into host cells $[28,29]$, which are mainly involved in the regulation of pathological processes, such as inflammatory response, oxidative stress and lipid metabolism of host cells [30]. In addition, studies have found that Enterobacter cloacae can activate inflammatory and autophagy pathways, triggering metabolic disorders in the body, resulting in heterotopic calcification, namely the formation of atherosclerotic plaques [31]. These suggest that the formation of Randall's calcium plaques as ectopic calcification of the kidney may also be regulated by Enterobacter cloacae. To the best of our understanding, we are the first to discover Enterobacter cloacae existing in pelvis urine of patients with $\mathrm{CaOx}$ stones. The mechanism of Enterobacter cloacae inducing $\mathrm{CaOx}$ stones still remains unclear.

To further investigate the role Enterobacter cloacae plays in $\mathrm{CaOx}$ stones formation, we conducted Whole Genome Sequencing of Enterobacter cloacae. Bacteria act on the receptor cells through their virulence factors, or their metabolites, extracellular vesicles. Virulence factors are components of bacterial virulence, and they act in two forms: invasiveness and toxin. By comparing the gene coding protein sequence of the Enterobacter cloacae with the amino acid sequence in the VFDB (Virulence Factors of Pathogenic Bacteria) database, we found that flagellum, lipopolysaccharide, aeromycin and urease had high homology, among which flagellum-related protein abundance was the highest. Flagellin is an important scaffold of bacterial movement, dynamic element and virulence factor. Flagellin is the only specific ligand of Toll-like receptor 5, the host membrane protein receptor, which has strong immunogenicity. As a unique structural protein of bacteria, flagellin is also one of the most abundant proteins in bacteria. As a virulence factor, flagellin can promote the invasion of pathogenic bacteria. Therefore, flagellin is a key target for host immune monitoring. When pathogenic bacteria invade the host, the natural immune receptor TLR5 can easily recognize flagellin, thus inducing MAPK- and NF- $\mathrm{kB}$-mediated inflammatory response [32]. Some investigators have reported that Flagellin could accelerate $\mathrm{CaOx}$ deposits by promoting its crystallization, growth and aggregation [12]. What's more, flagellin could also facilitate $\mathrm{Ca}^{2+}$ influx via calcium cellular membrane channel [33]. However, the association of flagellin with $\mathrm{CaOx}$ deposits has been only superficially studied, more studies should be applied to explore the mechanism of flagellin inducing $\mathrm{CaOx}$ deposits.

KEGG and GO analysis revealed Enterobacter cloacae may play important roles in ion binding and signaling transduction. Cherng et al. found that bacterial infection contributed to the formation of $\mathrm{CaOx}$ deposits via calcium-related ion channels after injected bacteria [34]. Cell signal transduction is a process in which cells experience the stimulation of information molecules through cellular membrane or intracellular receptors, and then transform by intracellular signal transduction system, thus affecting the biological functions of cells. It broadly existed in many biological processes. There are many signaling transduction pathways involved in $\mathrm{CaOx}$ stones formation. The P-38/JNK MAPK transduction pathway is turned on in the process of $\mathrm{CaOx}$ stone formation [35].

To validate our previous claims, we have conducted in vivo experiments. We assessed the apoptosis condition and crystal depositions of kidneys between rats with different treatments. Pre-injected with Enterobacter cloacae could apparently accelerate the apoptosis and crystal depositions of metabolic disorders rats induced by Glyoxylic Acid. While injected Glyoxylic or Enterobacter cloacae alone did not make any difference in apoptosis condition and crystal depositions comparing to control groups. What's more, the expression of IL-6, MCP-1, BMP2 and OPN was higher in rats injected with Glyoxylic Acid or Enterobacter cloacae than control. Treated with both Enterobacter cloacae and Glyoxylic Acid could enhance the increases of these proteins. It has been previously demonstrated that IL-6 and MCP-1 indicated a close relationship between inflammation 
and kidney stones, while our previous research has confirmed that osteogenic transformation-related proteins BMP2 and OPN played important roles in stone formation $[36,37]$. Thus, we could presume that pre-existence of Enterobacter cloacae will enhance the effects of metabolic disorders in $\mathrm{CaOx}$ stones formation through inflammation process and osteogenic transformation. However, the process of $\mathrm{CaOx}$ stones formation is complex, bacteria themselves might cause infection that induce inflammatory response and macrophages recruitment [38]. We cannot be sure that Enterobacter cloacae is the special regulator of $\mathrm{CaOx}$ stones formation via inflammation and osteogenic transformation, or if this is a general phenomenon with kidney infection. But our study is just a preliminary exploration based on a finding that there exist different microbiota in stone sides and non-stone sides pelvis urine of patients with unilateral $\mathrm{CaOx}$ stones and our in vivo experiments demonstrates that Enterobacter cloacae has associations with $\mathrm{CaOx}$ stones formation, while the specific mechanism still needs to be further explored. In addition, we look forward to future studies which can explore the relationship of other bacteria with stone formation.

In summary, our results revealed that the occurrence of bacteria is considerably correlated with $\mathrm{CaOx}$ stones formation. Enterobacter cloacae and its flagellin are supposed to have effects on $\mathrm{CaOx}$ stone formation through ion binding and signaling transduction. In vivo experiments we conducted have identified that Enterobacter cloacae could accelerate the stone formation of rats with metabolic disorders. However, we just hypothesized the possible process of $\mathrm{CaOx}$ stone formation; further studies are required to explore the detailed mechanism of Enterobacter cloacae and its Flagellin inducing $\mathrm{CaOx}$ stone formation. What's more, more patients should be recruited in our studies to increase the trustworthiness of our outcomes.

\section{Conclusion}

Microbiome colonized in kidney are associated with kidney stone formation. Enterobacter cloacae colonized in kidney can accelerate $\mathrm{CaOx}$ stone formation, while the concrete mechanism still needs more explorations.

Supplementary Information The online version contains supplementary material available at https://doi.org/10.1007/s00240-022-01311-8.

\section{Declarations}

Conflict of interest The authors declare that there are no conflict of interests.
Open Access This article is licensed under a Creative Commons Attribution 4.0 International License, which permits use, sharing, adaptation, distribution and reproduction in any medium or format, as long as you give appropriate credit to the original author(s) and the source, provide a link to the Creative Commons licence, and indicate if changes were made. The images or other third party material in this article are included in the article's Creative Commons licence, unless indicated otherwise in a credit line to the material. If material is not included in the article's Creative Commons licence and your intended use is not permitted by statutory regulation or exceeds the permitted use, you will need to obtain permission directly from the copyright holder. To view a copy of this licence, visit http://creativecommons.org/licenses/by/4.0/.

\section{References}

1. Scales CD Jr, Smith AC, Hanley JM, Saigal CS (2012) Prevalence of kidney stones in the United States. Eur Urol 62(1):160-165

2. Zeng G, Mai Z, Xia S, Wang Z, Zhang K, Wang L, Long Y, Ma J, Li Y, Wan SP, Wu W, Liu Y, Cui Z, Zhao Z, Qin J, Zeng T, Liu Y, Duan X, Mai X, Yang Z, Kong Z, Zhang T, Cai C, Shao Y, Yue Z, Li S, Ding J, Tang S, Ye Z (2017) Prevalence of kidney stones in China: an ultrasonography based cross-sectional study. BJU Int 120(1):109-116

3. Gambaro G, Croppi E, Bushinsky D, Jaeger P, Cupisti A, Ticinesi A, Mazzaferro S, D'Addessi A, Ferraro PM (2017) The risk of chronic kidney disease associated with urolithiasis and its urological treatments: a review. J Urol 198(2):268-273

4. Zisman AL (2017) Effectiveness of treatment modalities on kidney stone recurrence. Clin J Am Soc Nephrol 12(10):1699-1708

5. Howles SA, Thakker RV (2020) Genetics of kidney stone disease. Nat Rev Urol 17(7):407-421

6. Mehta M, Goldfarb DS, Nazzal L (2016) The role of the microbiome in kidney stone formation. Int J Surg 36(Pt D):607-612

7. Fuster DG, Morard GA, Schneider L, Mattmann C, Lüthi D, Vogt B, Dhayat NA (2020) Association of urinary sex steroid hormones with urinary calcium, oxalate and citrate excretion in kidney stone formers. Nephrol Dial Transplant. https://doi.org/10.1093/ndt/ gfaa360

8. Coe FL, Worcester EM, Evan AP (2016) Idiopathic hypercalciuria and formation of calcium renal stones. Nat Rev Nephrol 12(9):519-533

9. Durbin JM, Stroup SP, Altamar HO, L'Esperance JO, Lacey DR, Auge BK (2012) Genitourinary abnormalities in an asymptomatic screening population: findings on virtual colonoscopy. Clin Nephrol 77(3):204-210

10. Espinosa-Ortiz EJ, Eisner BH, Lange D, Gerlach R (2019) Current insights into the mechanisms and management of infection stones. Nat Rev Urol 16(1):35-53

11. Huang ZQ, Qiu JX, Li J, Dp Xu, Liu Q (2020) Exploration of microbial diversity based on 16S rRNA gene sequence analysis. Acta Microbiol Sin 61(5):1044-1063

12. Kanlaya R, Naruepantawart O, Thongboonkerd V (2019) Flagellum is responsible for promoting effects of viable escherichia coli on calcium oxalate crystallization, crystal growth, and crystal aggregation. Front Microbiol 10:2507

13. Chutipongtanate S, Sutthimethakorn S, Chiangjong W, Thongboonkerd V (2013) Bacteria can promote calcium oxalate crystal growth and aggregation. J Biol Inorg Chem 18(3):299-308

14. Dornbier RA, Bajic P, Van Kuiken M, Jardaneh A, Lin H, Gao X, Knudsen B, Dong Q, Wolfe AJ, Schwaderer AL (2020) The microbiome of calcium-based urinary stones. Urolithiasis 48(3):191-199 
15. Kachroo N, Lange D, Penniston KL, Stern J, Tasian G, Bajic P, Wolfe AJ, Suryavanshi M, Ticinesi A, Meschi T, Monga M, Miller AW (2021) Standardization of microbiome studies for urolithiasis: an international consensus agreement. Nat Rev Urol 18(5):303-311

16. Bolyen E, Rideout JR, Dillon MR, Bokulich NA, Abnet CC, AlGhalith GA, Alexander H, Alm EJ, Arumugam M, Asnicar F, Bai Y, Bisanz JE, Bittinger K, Brejnrod A, Brislawn CJ, Brown CT, Callahan BJ, Caraballo-Rodríguez AM, Chase J, Cope EK, Da Silva R, Diener C, Dorrestein PC, Douglas GM, Durall DM, Duvallet C, Edwardson CF, Ernst M, Estaki M, Fouquier J, Gauglitz JM, Gibbons SM, Gibson DL, Gonzalez A, Gorlick K, Guo J, Hillmann B, Holmes S, Holste H, Huttenhower C, Huttley GA, Janssen S, Jarmusch AK, Jiang L, Kaehler BD, Kang KB, Keefe CR, Keim P, Kelley ST, Knights D, Koester I, Kosciolek T, Kreps J, Langille MGI, Lee J, Ley R, Liu YX, Loftfield E, Lozupone C, Maher M, Marotz C, Martin BD, McDonald D, McIver LJ, Melnik AV, Metcalf JL, Morgan SC, Morton JT, Naimey AT, Navas-Molina JA, Nothias LF, Orchanian SB, Pearson T, Peoples SL, Petras D, Preuss ML, Pruesse E, Rasmussen LB, Rivers A, Robeson MS 2nd, Rosenthal P, Segata N, Shaffer M, Shiffer A, Sinha R, Song SJ, Spear JR, Swafford AD, Thompson LR, Torres PJ, Trinh P, Tripathi A, Turnbaugh PJ, Ul-Hasan S, van der Hooft JJJ, Vargas F, Vázquez-Baeza Y, Vogtmann E, von Hippel M, Walters W, Wan Y, Wang M, Warren J, Weber KC, Williamson CHD, Willis AD, Xu ZZ, Zaneveld JR, Zhang Y, Zhu Q, Knight R, Caporaso JG (2019) Reproducible, interactive, scalable and extensible microbiome data science using QIIME 2. Nat Biotechnol 37(8):852-857

17. Callahan BJ, McMurdie PJ, Rosen MJ, Han AW, Johnson AJ, Holmes SP (2016) DADA2: High-resolution sample inference from Illumina amplicon data. Nat Methods 13(7):581-583

18. Bokulich NA, Kaehler BD, Rideout JR, Dillon M, Bolyen E, Knight R, Huttley GA, Gregory Caporaso J (2018) Optimizing taxonomic classification of marker-gene amplicon sequences with QIIME 2's q2-feature-classifier plugin. Microbiome 6(1):90

19. Kõljalg U, Nilsson RH, Abarenkov K, Tedersoo L, Taylor AF, Bahram M, Bates ST, Bruns TD, Bengtsson-Palme J, Callaghan TM, Douglas B, Drenkhan T, Eberhardt U, Dueñas M, Grebenc T, Griffith GW, Hartmann M, Kirk PM, Kohout P, Larsson E, Lindahl BD, Lücking R, Martín MP, Matheny PB, Nguyen NH, Niskanen T, Oja J, Peay KG, Peintner U, Peterson M, Põldmaa K, Saag L, Saar I, Schüßler A, Scott JA, Senés C, Smith ME, Suija A, Taylor DL, Telleria MT, Weiss M, Larsson KH (2013) Towards a unified paradigm for sequence-based identification of fungi. Mol Ecol 22(21):5271-5277

20. Lozupone C, Knight R (2005) UniFrac: a new phylogenetic method for comparing microbial communities. Appl Environ Microbiol 71(12):8228-8235

21. Ramette A (2007) Multivariate analyses in microbial ecology. FEMS Microbiol Ecol 62(2):142-160

22. Zaura E, Keijser BJ, Huse SM, Crielaard W (2009) Defining the healthy "core microbiome" of oral microbial communities. BMC Microbiol 9:259

23. Flannigan R, Choy WH, Chew B, Lange D (2014) Renal struvite stones-pathogenesis, microbiology, and management strategies. Nat Rev Urol 11(6):333-341

24. Huang WY, Chen YF, Chen SC, Lee YJ, Lan CF, Huang KH (2012) Pediatric urolithiasis in Taiwan: a nationwide study, 1997 2006. Urology 79(6):1355-1359
25. Barr-Beare E, Saxena V, Hilt EE, Thomas-White K, Schober M, Li B, Becknell B, Hains DS, Wolfe AJ, Schwaderer AL (2015) The interaction between Enterobacteriaceae and Calcium Oxalate deposits. PLoS ONE 10(10):e0139575

26. Tasian GE, Jemielita T, Goldfarb DS, Copelovitch L, Gerber JS, Wu Q, Denburg MR (2018) Oral antibiotic exposure and kidney stone disease. J Am Soc Nephrol 29(6):1731-1740

27. Song J, Min L, Wu J, He Q, Chen F, Wang Y (2021) Response of the microbial community to phosphate-solubilizing bacterial inoculants on Ulmus chenmoui Cheng in Eastern China. PLoS ONE 16(2): 0247309

28. Dai PF, Du Y (2020) Impact of NDM-1 on pathogenicity and fitness of enterobacter cloacae NDM-1. Prog in Microbiol Immunol 48(03):84-88

29. Colonne PM, Winchell CG, Voth DE (2016) Hijacking host cell highways: manipulation of the host actin cytoskeleton by obligate intracellular bacterial pathogens. Front Cell Infect Microbiol 6:107

30. Barnes AI, Paraje MG, del Paolo CB, Albesa I (2001) Molecular properties and metabolic effect on blood cells produced by a new toxin of Enterobacter cloacae. Cell Biol Toxicol 17(6):409-418

31. Hirano S, Ohkawa M, Nakajima T, Orito M, Sugata T, Hisazumi $\mathrm{H}$ (1985) Renal infections and implicated urinary stone formation. Hinyokika Kiyo 31(8):1387-1391

32. Haney C, Urbach J, Ausubel F (2014) Differences and similarities: Innate immunity in plants and animals. Biochemist 36:40-44

33. Chen YL, Chen YS, Lin $\mathrm{HH}$, Chan $\mathrm{CW}$, Chen SC, Chen $\mathrm{CH}$ (2007) Immunostimulatory flagellin from Burkholderia pseudomallei effects on an increase in the intracellular calcium concentration and up-regulation of TNF-alpha by mononuclear cells. Microbiol Immunol 51(1):81-86

34. Cherng JH, Hsu YJ, Liu CC, Tang SH, Sartika D, Chang SJ, Fan GY, Wu ST, Meng E (2019) Activities of $\mathrm{Ca}(2+)$-related ion channels during the formation of kidney stones in an infection-induced urolithiasis rat model. Am J Physiol Renal Physiol 317(5):F1342-f1349

35. Khan SR (2013) Reactive oxygen species as the molecular modulators of calcium oxalate kidney stone formation: evidence from clinical and experimental investigations. J Urol 189(3):803-811

36. Jia Z, Wang S, Tang J, He D, Cui L, Liu Z, Guo B, Huang L, Lu Y, Hu H (2014) Does crystal deposition in genetic hypercalciuric rat kidney tissue share similarities with bone formation? Urology 83(2):509.e507-514

37. Jia Z, Wang S, He D, Cui L, Lu Y, Hu H, Qin B, Zhao Z (2015) Role of calcium in the regulation of bone morphogenetic protein 2 , runt-related transcription factor 2 and Osterix in primary renal tubular epithelial cells by the vitamin D receptor. Mol Med Rep 12(2):2082-2088

38. Khan SR, Canales BK, Dominguez-Gutierrez PR (2021) Randall's plaque and calcium oxalate stone formation: role for immunity and inflammation. Nat Rev Nephrol 17(6):417-433

Publisher's Note Springer Nature remains neutral with regard to jurisdictional claims in published maps and institutional affiliations. 